\title{
El Profesor Posmoderno de Ciencias Sociales: Un Modelo de Buenas Prácticas en Educación Patrimonial
}

\section{The Postmodern Teacher of Social Sciences: A Model of Good Practices in Heritage Education}

\author{
Laura Lucas ${ }^{1 *}$ \\ Emilio José Delgado-Algarra ${ }^{2}$ \\ ${ }^{1}$ Universidad de Valladolid, España \\ ${ }^{2}$ Universidad de Huelva, España
}

\begin{abstract}
En el presente artículo se lleva a cabo un estudio de caso apoyado en la Teoría Fundamentada con la finalidad de definir un catálogo de buenas prácticas educativas del profesorado innovador de Ciencias Sociales de Educación Secundaria Obligatoria, en relación con el patrimonio como contenido pedagógico para la formación de una ciudadanía crítica, participativa e igualitaria y analizar en profundidad un caso cercano a un modelo deseable, que hemos denominado profesor posmoderno. El análisis se desarrolla mediante un proceso de doble triangulación el cual, desde un enfoque multimetódico, permite contrastar la intersubjetividad de nuestro proceso de investigación cualitativa. Así se destacan las convergencias y divergencias entre las concepciones del docente y su práctica bajo el paraguas del sistema de categorías. A raíz de la percepción que los estudiantes tienen del desarrollo de las clases y la interpretación que el docente hace de dichas percepciones reforzamos la validez y fiabilidad de las interpretaciones de la investigadora. Entre las conclusiones está la importancia de que el docente de Ciencias Sociales conecte su formación académica con la práctica profesional para la mejora educativa; incluyendo la educación patrimonial como factor fundamental para formar una ciudadanía comprometida con la realidad.
\end{abstract}

Descriptores: Enseñanza; Estudio de caso; Ciudadanía; Patrimonio cultural; Innovación educacional.

From the point of view of a critical didactic, school content cannot be understood as an element disconnected from the relevant socio-environmental problems. In this way, define a catalog of good educational practices of the innovative teachers of Social Sciences of Compulsory Secondary Education, in relation to heritage as a pedagogical content for the formation of a critical, participatory and egalitarian citizenship; and analyze in depth a case close to a desirable model; a case study based on the Grounded Theory is carried out. The analysis is developed through a double triangulation process which, from a multimethod approach, allows us to contrast intersubjectivity of our qualitative research process. We highlight the convergences and divergence between the teacher's conceptions and their practice under the umbrella of the category system. As a result of the perception that the students have of the development of the classes and the interpretation that the teacher makes of these perceptions, we reinforce the validity and reliability of the interpretations of the researcher. One of the most important conclusions is the importance of the teacher to connect their academic training with professional practice for educational improvement; including patrimonial education as a fundamental factor to form a citizenship committed to reality.

Keywords: Teaching, case studies; Citizenship; Cultural heritage; Educational innovation.

*Contacto: laura.lucas.palacios@uva.es

ISSN: 1696-4713

www.rinace.net/reice/

revistas.uam.es/reice
Recibido: $\quad 19$ de abril 2019

$1^{\text {a }}$ Evaluación: 20 de mayo 2019

$2^{\text {a }}$ Evaluación: 31 de mayo 2019

Aceptado: $\quad 5$ de junio 2019 


\section{Introducción}

Desde el planteamiento de una escuela entendida como motor de cambio social y en un contex to de crecimiento de los ambientes de pluralidad cultural, se hace necesario hoy más que nunca, que nuestros jóvenes aprendan a participar en la vida pública y asuman su compromiso como parte de la misma.

Por ello, es clave la inclusión de la educación patrimonial en el aula, como una de las fuentes de la educación ciudadana. Lucas (2018) establece tres contenidos que conectan ambas áreas: identidad, vinculado a una ciudadanía como sentimiento; conservación, vinculado a una ciudadanía como estatus; y gestión, vinculado a una ciudadanía como práctica. Por otro lado, la identidad puede plantearse a tres niveles: individual, sociocolectiva o intercultural. No obstante, como destacan Monteagudo y Oliveros (2016), a la hora de enseñar aspectos patrimoniales, resulta habitual que se seleccione un conocimiento patrimonial ligado al patrimonio histórico-artístico, por el sesgo disciplinar que tradicionalmente le ha acompañado hasta los años ochenta. Momento en el que se empezó a hablar de patrimonio cultural (Martín Cáceres, 2012),

En líneas generales, se presentan algunos de los resultados más relevantes de una investigación que se enmarca en una Tesis doctoral vinculada con el proyecto de Investigación EPITEC "Educación Patrimonial para la Inteligencia Territorial y Emocional de la Ciudadanía” (EDU2015-67953-P) financiado por el Ministerio de Economía y Competitividad del Gobierno de España, con la cofinanciación de los fondos FEDER de la Unión Europea. Para la investigación, seleccionamos como caso de estudio un profesor de Ciencias Sociales de Educación Secundaria, modelo de buenas prácticas, registrando los datos obtenidos mediante diferentes técnicas de recogida de información, como grabaciones de aula, entrevistas en profundidad y grupos de discusión.

\section{El profesor posmoderno en la enseñanza de las Ciencias Sociales}

Como afirman Porlán y Rivero (1998) y Blömeke y otros (2016) el conocimiento profesional deseable del profesorado no puede reducirse a la transmisión de un saber académico. El docente, como agente educativo responsable de la planificación y siendo el tomador de las decisiones más importantes que ocurren en el aula:

- Es un sujeto subjetivo, reflexivo, racional, que toma decisiones, emite juicios, tiene creencias y genera rutinas propias de su desarrollo profesional (Ancess, Barnet y Allen, 2007; Elliot, 2010; Rathgen, 2006).

- Sus pensamientos son los que guían y orientan su conducta (Arthur, Davies y Hahn, 2008; Delgado-Algarra, 2015; Escudero y Martínez Domínguez, 2016; Eurydice, 2012; García Pérez, 2006; Molina Girón, 2013).

Por ello, tomando como referencia los planteamientos de una didáctica crítica en la que la educación patrimonial es un contenido fundamental para construir una ciudadanía activa, democrática, reflexiva y responsable, se plantea como necesario que el profesorado de Ciencias Sociales ponga en marcha una didáctica de corte crítico y que apueste firmemente por una educación democrática y patrimonial (figura 1). 


\section{ENSEÑANZA CIENCIAS SOCIALES}

Definen la

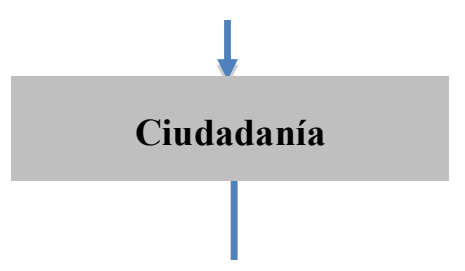

A partir de la

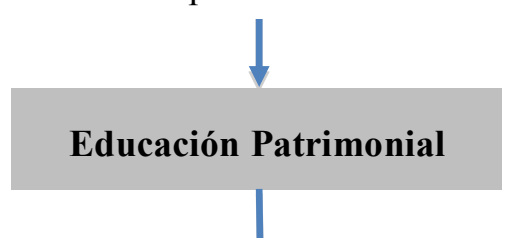

Se adquiere

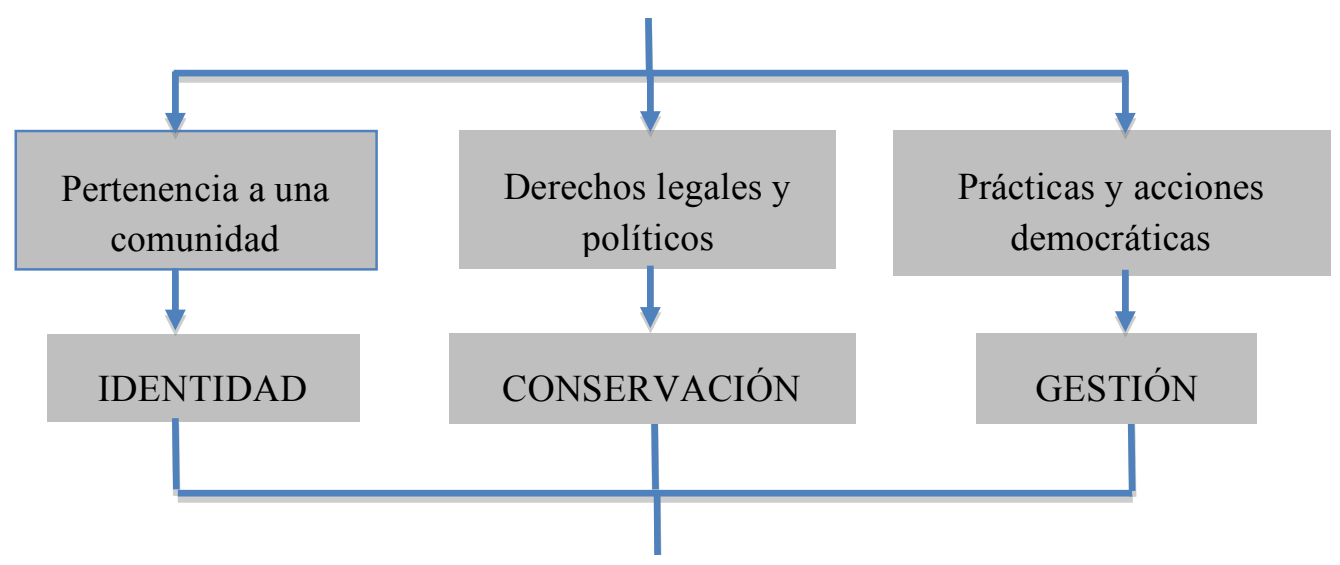

Posibilitando la

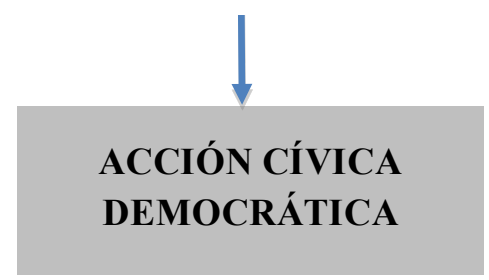

Figura 1. Propuesta para una educación democrática de la ciudadanía a partir de la educación patrimonial

Fuente: Elaboración propia.

Para que esto ocurra, como afirma Navarro (2012), no basta con cambiar objetivos o la selección de contenidos, sino que se debe cambiar la cultura escolar, la del profesorado, entendida como mediador, y la del alumnado, entendido como protagonista de los cambios. Así, el profesorado de Ciencias Sociales debe asegurar una democracia deliberativa y participativa acorde con la realidad de los jóvenes, por encima de los datos aislados y de la simple trasmisión de contenidos (De Alba, 2007; Sobejano y Torres, 2010). 
Efland, Freedman y Stuhr (2003), Calaf (2009) y De Castro (2016), desde el ámbito de la expresión artística, aportan un nuevo modelo docente: el profesor posmoderno, que se caracteriza por adoptar un enfoque curricular interdisciplinario y multidisciplinario, en el que alumnado y profesorado trabajan juntos para analizar y comprender el mundo desde el presente. Continuando con dichos autores, y desde el área de Ciencias Sociales, Geografía e Historia, es necesario apostar por relatos alternativos a los propuestos desde las disciplinas de referencia, asumiendo que existen diferentes interpretaciones de los acontecimientos históricos que permiten comprender la realidad.

Con todo ello, consideramos como referente de profesor de Ciencia Sociales deseable a un profesor que parte de problemas relevantes para comprender tanto el presente como el pasado, utilizando el patrimonio como contenido integrador para afrontar estos problemas como ciudadanos críticos. Con una metodología participativa, indagativa y flexible, que se ve a sí mismo como un mediador del proceso de enseñanza-aprendizaje, donde el alumnado es el verdadero protagonista y que entiende la escuela como motor de cambio social donde confluyen críticamente el conocimiento cotidiano, escolar y científico. Es lo que nosotros hemos llamado el profesor posmoderno.

\section{Método}

La finalidad de esta investigación se centra en definir un catálogo de buenas prácticas educativas del profesorado innovador de ESO, en relación con el patrimonio como contenido pedagógico para la formación de una ciudadanía crítica, participativa e igualitaria; y analizar en profundidad un caso cercano a un modelo deseable. De forma que, nos planteamos como problema principal de investigación ¿Qué y cómo enseña el profesorado innovador educación para la ciudadanía utilizando el patrimonio como contenido pedagógico en Ciencias Sociales, Geografía e Historia en Educación Secundaria Obligatoria? En líneas generales, la investigación presentada se plantea desde un enfoque cualitativo destinado a comprender de manera global, descriptiva e interpretativa nuestra problemática, analizando la realidad desde una perspectiva holística y dinámica, y a través diversas técnicas (Eisner, 1998; Rossman y Rallis, 1989). Inicialmente, estamos ante un diseño de investigación proyectado cuyos fundamentos y estructura se plantean previamente a la puesta en marcha de la investigación (Martínez Rodríguez, 2013). Sin embargo, en consonancia con la Teoría Fundamentada de Glaser y Strauss donde se establece que, desde un enfoque inductivo, la inmersión de los datos sirve como una de las referencias para construir el diseño teórico (Páramo, 2015); a medida que avanzaba la investigación, la estructura y los instrumentos de análisis se fueron perfilando y matizando.

\section{Participantes}

El muestreo fue no probabilístico por criterio, buscando que los participantes representaran cualitativamente el grupo de referencia; por tanto, para la selección de los mismos se fijaron como criterios ser docentes de Ciencias Sociales, Geografía e Historia de Educación Secundaria Obligatoria y que participaran activamente en proyectos de innovación educativa bien sea a nivel de centro o a través de programas universitarios. El acercamiento a estos docentes fue a través de los programas de innovación educativa que tiene la universidad de Sevilla y que permite trazar redes con docentes que apuestan por 
la innovación educativa en su praxis. Finalmente fueron 10 los docentes que cumplían estos requisitos.

Tras el análisis de estos cuestionarios, seis de los/as participantes respondían al perfil que considerábamos deseable según nuestra hipótesis de progresión (cuadro 1). Dicha hipótesis está secuenciada en tres niveles de menor a mayor complejidad del concepto de patrimonio y de ciudadanía y de su integración en la praxis educativa. Para su diseño, tomamos como referencia trabajos como los de Cuenca (2003) y Matozzi y Ávila (2009), en relación con el patrimonio; y como los de García-Pérez (2002), Schulz (2012) y Schugurensky (2012), en relación con la educación para la ciudadanía.

Cuadro 1. Nivel deseable de la hipótesis de progresión del conocimiento profesional del profesorado para enseñar educación para la ciudadanía a través de la educación patrimonial

\begin{tabular}{l} 
NIVEL DESEABLE \\
\hline Concepción del patrimonio desde una perspectiva simbólico-identitaria de una sociedad \\
globalizada. \\
Visión integradora de las manifestaciones patrimoniales desarrollando actitudes ciudadanas \\
que implican su conservación activa e intervencionista y su compromiso personal. \\
Tratamiento del patrimonio totalmente integrado en el proceso educativo como contenido de \\
enseñanza en el desarrollo de actitudes ciudadanas en procesos democráticos reales. \\
Diseño de propuestas de carácter multidireccional con la participación activa y comprometida \\
del alumno/a en proyectos de conservación patrimonial, elaborados en torno a problemas \\
socialmente relevantes. \\
Planificación integradora de conceptos, procedimientos y actitudes. \\
Diseño de propuestas desde un planteamiento coeducativo a partir de la educación patrimonial. \\
\hline Fuente: Elaboración propia, basado en los trabajos mencionados en el texto.
\end{tabular}

Tras la revisión de las entrevistas, se lleva a cabo la selección del caso de estudio representado por el docente VPG, profesor de Ciencias Sociales, Geografía e Historia de los cursos $1^{\circ}-4^{\circ}$ de Educación Secundaria Obligatoria en el centro IEs Castillo de Luna de La Puebla de Cazalla (Sevilla, España).

\section{Sistema de categorías}

Para orientar el proceso de recogida y organización de la información y en base a múltiples fuentes como las mencionadas en el subapartado se diseña un sistema de tres categorías: enseñanza del patrimonio, enseñanza de ciudadanía, enseñanza de la educación para la ciudadanía a través del patrimonio. El diseño del sistema de categorías de basa en investigaciones como las de Cuenca (2002), Delgado-Algarra y Estepa (2016, 2017), Estepa (2013) y Lucas (2018), cuya estructura específica bebe de múltiples fuentes. En el cuadro 2 , se presentan categorías, subcategorías e indicadores del sistema, no incluyendo descriptores por motivos de espacio.

\section{Recogida de informaciones}

El empleo de técnicas cuantitativas como el uso de cuestionarios, no impide, en ningún momento, el análisis cualitativo de los datos obtenidos desde una "perspectiva de la educación caracterizada por consideraciones sistémicas, complejas y críticas” (Cuenca, 2002). Así pues, tras pasar un cuestionario inicial (Co) para la preselección del caso, los instrumentos de recogida son: 
- Entrevista exploratoria (E1): una entrevista semiestructurada está compuesta por 10 cuestiones relacionadas con el sistema presentado en el subapartado anterior.

- Observación de aula (O1): científica, directa y participante. Se registran doce sesiones de la asignatura Ciencias Sociales de un curso de $2^{\circ}$ de ESO, en un centro de secundaria. A lo largo de dichas sesiones se desarrolló el bloque temático "AlÁndalus y los reinos cristianos", establecido en la Orden de 17 marzo de 2015, por la que se desarrolla el currículo correspondiente a la Educación Secundaria Obligatoria en Andalucía.

- Grupo de discusión dicente (GD): En nuestro caso, los grupos de discusión que participan en esta investigación son los siguientes:

$\checkmark$ GD1. La Sevilla islámica.

$\checkmark$ GD2. El escudo de España y los reinos medievales.

$\checkmark$ GD3-4. La mujer cristiana en la Edad Media y La mujer musulmana en la Edad Media.

$\checkmark$ GD5. El camino de Santiago.

- Entrevista final docente (E2): más abierta que la entrevista exploratoria.

Se contó así con 24 archivos de audio de 1.920 minutos de grabación, que fueron transcritas en 846 páginas; así como 56 páginas del diario de observación de la investigadora y 16 plantillas de análisis de información de las entrevistas y los cuestionarios. Todo ello fue categorizado y analizado por el programa informático Atlas.ti.

En cuanto a la categorización de la información se realizó de la siguiente manera: en primer lugar, se especifica el instrumento, seguido del número de sesión o de grupo de discusión (O 1, GD4) y el número de anotación registrada en el programa Atlas.ti. Cuando el instrumento aplicado es la entrevista se especifica si es la entrevista inicial con E1 o si es la final con E2.

\section{Análisis de información}

El análisis de las informaciones, además de tener en cuenta el sistema de categorías, se configura tomando como referencia el modelo de doble triangulación de Delgado-Algarra y Estepa (2016); el cual, desde un enfoque multimetódico, permite contrastar la intersubjetividad y asegurar la credibilidad de nuestro proceso de investigación cualitativa. De este modo, con E1 y O1 se ponen de manifiesto las convergencias y divergencias existentes entre las concepciones del docente y su práctica bajo el paraguas del sistema de categorías. En relación con la segunda triangulación (GD-O1-E2), a raíz de la percepción que los estudiantes tienen del desarrollo de las clases y la interpretación que el docente hace de dichas percepciones. 
Cuadro 2. Sistema de categorías para el análisis de la praxis

\section{CATEGORÍAS}

Enseñanza del
patrimonio

SUBCATEGORÍAS

Concepto de patrimonio

Perspectiva simbólico-identitaria

Natural

Histórico

Tipología patrimonial

Artístico

Etnológico

Científico -Tecnológico

Holístico

Patrimonio sin interés educativo

Qué se enseña de educación patrimonial en CCSS

Contenidos históricos-artísticos

Justicia Social

Inteligencia Emocional

Clases expositivas

Cómo se enseña educación patrimonial en CCSS

Materiales específicos

Visitas

Aprendizaje basado en proyectos

\begin{tabular}{|c|c|c|}
\hline \multirow{9}{*}{$\begin{array}{l}\text { Enseñanza de } \\
\text { ciudadanía }\end{array}$} & Concepto de ciudadanía & $\begin{array}{l}\text { Adaptativa } \\
\text { Crítica }\end{array}$ \\
\hline & & Individual \\
\hline & & Comunitarista \\
\hline & Tipos de ciudadanía & Cosmopolita \\
\hline & & Ciudadanía Digital \\
\hline & & Democrática Radical \\
\hline & $\begin{array}{l}\text { Tipología de participación } \\
\text { ciudadana }\end{array}$ & $\begin{array}{l}\text { Ciudadano personalmente responsable } \\
\text { Ciudadano activista } \\
\text { Ciudadano orientado a la justicia }\end{array}$ \\
\hline & $\begin{array}{l}\text { Qué se enseña de ecuación } \\
\text { para la ciudadanía en } \\
\text { CCSS }\end{array}$ & $\begin{array}{l}\text { Derechos individuales y conocimientos } \\
\text { de las instituciones estatales y civiles } \\
\text { Principios cívicos } \\
\text { Identidades cívicas } \\
\text { Participación cívica }\end{array}$ \\
\hline & $\begin{array}{l}\text { Cómo se enseña educación } \\
\text { para la ciudadanía en } \\
\text { CCSS }\end{array}$ & $\begin{array}{l}\text { Compromiso del profesor/a } \\
\text { Materiales curriculares }\end{array}$ \\
\hline \multirow{3}{*}{$\begin{array}{l}\text { Enseñanza de la } \\
\text { educación para la } \\
\text { ciudadanía a través } \\
\text { del patrimonio }\end{array}$} & Qué enseñar & $\begin{array}{l}\text { Identidad } \\
\text { Conservación } \\
\text { Gestión }\end{array}$ \\
\hline & Cómo enseñar & $\begin{array}{l}\text { Integración anecdótica } \\
\text { Fuente de análisis } \\
\text { Imbricación plena }\end{array}$ \\
\hline & Para qué enseñar & $\begin{array}{l}\text { Visión culturalista } \\
\text { Visión práctica-conservacionista } \\
\text { Visión socio-crítica }\end{array}$ \\
\hline
\end{tabular}

Fuente: Elaboración propia. 


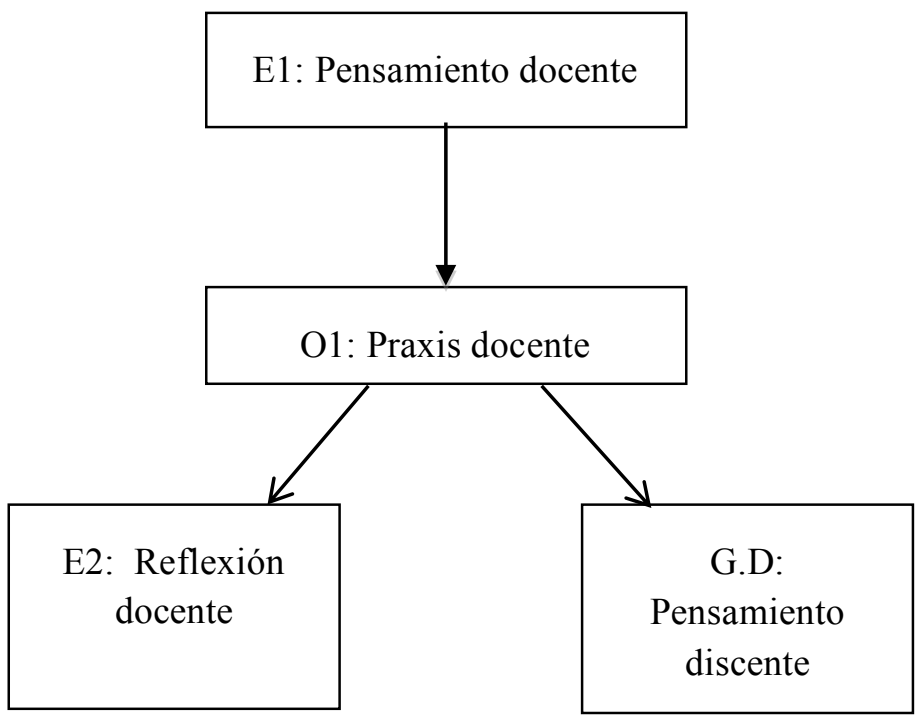

Figura 1. Esquema de doble triangulación utilizado en el estudio Fuente: Elaboración propia.

\section{Resultados}

Según Álvarez (2013), la superación de la quiebra teoría-práctica es siempre una cuestión delicada en la que no hay recetas. Sin embargo, las ideas que se recogen a continuación son pilares fundamentales en el proceso para este docente, y también pueden serlo para el profesorado que se lo proponga. Por ello, en los sucesivos apartados se irán revisando ideas que son clave en el proceso, argumentando sobre su relevancia y dando respuesta a las dificultades que surgen a la hora de llevarlas a cabo. Para realizar esta tarea con eficacia, en cada idea clave se desarrolla conjuntamente el análisis de los resultados y su discusión.

\subsection{Concepción de patrimonio y de ciudadanía}

Respondiendo al subproblema 1, ¿cuál es la concepción de ciudadanía y de patrimonio que tiene el profesor innovador y qué reflejo tiene en su práctica?; el profesor posmoderno tiene una concepción holística y simbólico-identitaria del patrimonio, así como una concepción de ciudadanía crítica, participativa, orientada a la justicia social y con una fuerte presencia de la tecnología en ella.

Para el docente del caso, los elementos patrimoniales forman parte de la identidad cultural de una comunidad y son una vía por la que la ciudadanía puede tomar conciencia de su papel como agentes activos en ella. Por tanto, para un profesor posmoderno, el patrimonio es un recurso inagotable y diverso que acoge no sólo las áreas específicamente humanas, sino también las científicas y tecnológicas (Cuenca, 2003; Estepa, Wamba y Jiménez, 2005). Entender el patrimonio y asumir su praxis y programación didáctica desde esta perspectiva, que nosotros hemos llamado holística, supone atribuir un papel plenamente activo a los procesos educativos y a sus protagonistas (Fontal, 2008).

$\mathrm{Al}$ revisar el registro de aula, vemos que así lo hace el profesor del caso de estudio, cuando trabaja con el grupo "La Sevilla islámica" qué elementos patrimoniales elegir para una posterior visita con el grupo-clase. 
Profesor: Muy bien, muy bien. Perfecto. Eso es muy interesante. Esa idea, ¿̇no? De que la ciudad tal y como la vemos es incomprensible sino pensamos en la historia que tiene detrás, ¿̇o?

Alumna: Tenemos que pensar antes en el después que en el ahora.

Profesor: Incluso una de vuestras conclusiones se podría enlazar con el título. Pensar la ciudad en el presente desde el pasado, pero sabiendo, que ese conocimiento, nos puede ayudar a entender cómo puede ser en el futuro. Me parece interesante que dijerais cosas con respecto a que tiene que ver con quiénes somos. Más que una ciudad, la ciudad son sus gentes, ¿̇no? (O5/Quotation: 5:2)

El docente entiende que la finalidad principal de su labor como profesional de la enseñanza es capacitar a su alumnado en los conocimientos y actitudes necesarias para su socialización. De forma que, en las distintas sesiones de aula, se trabaja temas que les permiten reflexionar sobre asuntos tan complejos como justicia social, responsabilidad cívica y respeto por las diferencias.

Profesor: Vosotros vais a estudiar a la mujer en la Edad Media y concretamente a la mujer andalusi (enfatiza "andalusí. Hace una pausa para recalcar este concepto) ... de andaluces" (vuelve a hacer otra pausa de tres segundos) ... ¿ ¿Puede ayudarnos el conocimiento de la mujer andalusí para comprender a la mujer musulmana actual?

Puede que el conocimiento del pasado nos ayude a comprender el presente, a saber, por qué algunas mujeres musulmanas llevan velo actualmente ¿̇ale?

Pero lo primero que tenemos que poner en cuestión es la idea de la mujer musulmana. Como si sólo hubiera una porque eso no es así, ¿vale? (O1 Quotation: 1:90)

No obstante, como indicábamos anteriormente, por encima de todo, para un profesor posmoderno, el concepto de ciudadanía tiene que estar en el plano de la acción. Por ello, un rasgo dominante en la praxis de este profesor es la utilización del debate e implicar al alumnado para que participe activamente, tanto en expresar sus opiniones como en la búsqueda de los contenidos. Por lo tanto, su praxis, se sitúa en la línea de Marí Ytarte, Moreno e Hipólito (2016), quienes defienden que la educación para la ciudadanía significa la voluntad de hacer partícipes a todos los sujetos de la educación del proceso de toma de decisiones y del aprendizaje de la vida en sociedad.

Profesor: Pero a mí me parece más importante primero saber qué es lo que queréis saber, ¿̇no? ¿Vosotros qué queréis investigar? ¿Cuál es el problema? (O2 Quotation: 2:2)

\subsection{Qué enseñar}

Respondiendo al subproblema 2, ¿qué enseña el profesor innovador de educación patrimonial y qué enseña de educación para la ciudadanía en Ciencias Sociales, Geografía e Historia?; el profesor posmoderno a través de un proceso de indagación basado en los referentes patrimoniales, busca que su alumnado comprenda conceptos relevantes del plano social, histórico, científico y ambiental, dando respuesta al planteamiento de problemas actuales.

En coherencia con lo manifestado en la entrevista inicial y en la praxis, nuestro docente tiene un tratamiento interdisciplinar del patrimonio. A través de los proyectos que se desarrollan en torno a los referentes patrimoniales, intenta que su alumnado desarrolle un pensamiento crítico que le permita dar respuesta a problemas actuales relacionados con la organización económica, política, social y cultural del presente; pero siempre comparando presente-pasado. 
Por ello, el profesor posmoderno, según De Castro (2016), entiende la educación desde su capacidad transformadora y cuestiona "la educación exclusivamente reproductiva, cognitivista, orientada únicamente al conocimiento intelectual-racional” (Barragán Rodríguez, 2005, p. 56). Este modelo de profesor tiene muy presente que el fin último de todo sistema educativo es la formación de una ciudadanía democrática y como tal, la participación y el compromiso social son los dos comportamientos básicos que debe de aprender su alumnado. Y así lo hace nuestro docente. Problemas tan actuales como la independencia de Cataluña, la violencia machista, la discriminación de género, la sociedad de consumo, el empleo de los recursos que hace el turismo insostenible o el terrorismo islámico están perfectamente enlazadas con los contenidos didácticos de los temas curriculares.

Profesor: España es un país que históricamente se compone de reinos, de regiones, de nacionalidades diferentes, cada una de las cuales tiene su propio símbolo. Cuando se escogió ese escudo lo que se quiso representar es esta diferencia... Los diferentes reinos caben dentro de un mismo país.

Esa idea de que en España cabemos todos es muy interesante para entender lo que está pasando actualmente. Por ejemplo, en Cataluña. (O2 Quotation: 2:46)

Profesor: Hay montones de leyendas que han servido para que la gente, sobre todo en la Edad Media -porque era cuando más devota era-quisiera hacer ese peregrinaje, ese viaje... $Y$ en el camino se iban dejando el dinero y generando riqueza. Todavía hoy día.

Alumno: Para hacer el camino tienes que pagar, ¿̇no?

Profesor: Tienes que comer y hay que beber, hay que alojarse, hay que comprar recuerdos... (O13 Quotation: 13:10)

Fontal (2006) asegura que el patrimonio permite investigar sobre los distintos tiempos de la cultura: la cultura del presente, la cultura del pasado, la cultura desde el presente, la cultura desde el pasado. Por tanto, la educación patrimonial posibilita al alumnado poder configurar una base cultural sólida para el futuro, como así nos lo confirmaron el 100\% de los estudiantes que participaron en los grupos de discusión:

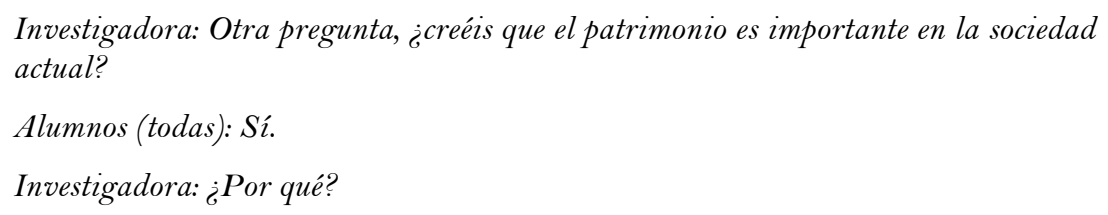

Con todo ello, coincidimos con Calaf (2009) cuando defiende que la educación patrimonial y la educación para la ciudadanía que imparte el profesor posmoderno debe recuperar las propuestas de educación experiencial dadas por Dewey, usando el juego y despertando el interés como estrategias de conocimiento, y las presentadas por Freire (1975)-situaciones de aprendizaje en torno a problemas de la vida real que el alumnado debe resolver a partir del patrimonio-. Llegamos así al siguiente punto.

\subsection{Cómo enseñar}

Respondiendo al subproblema 3, ¿cómo enseña el profesorado innovador educación patrimonial y cómo enseña educación para la ciudadanía en Ciencias Sociales, Geografía e Historia?; consideramos que el profesor posmoderno, tiene que entender la educación patrimonial como el eje transdisciplinar de la educación. Tanto en la praxis como en el 
pensamiento del docente existe una imbricación plena entre el patrimonio, los contenidos de Ciencias Sociales y la realidad socionatural del alumnado.

La didáctica del patrimonio no constituye un fin en sí mismo, sino que se integra en el proceso educativo. En palabras de De Castro (2016), "el patrimonio es la piedra filosofal de la educación. Todo lo que toca se convierte en una línea de trabajo tan intensa como hermosa. La educación patrimonial es la alquimia que hace esto posible" (p.455). Lo mismo ocurre con la educación para la ciudadanía que, como ya hemos dicho anteriormente y a nuestro modo de ver, debe ser el fin último de todo sistema educativo y, por tanto, un eje transversal en todas las materias educativas.

Para ello, el docente adopta el Aprendizaje Basado en Proyectos como su metodología de trabajo. Una metodología que permite que las materias se desvanezcan, las clases dejen de ser exclusivamente magistrales y los estudiantes sean los protagonistas de su propio aprendizaje, realizando tareas que tienen sentido y que son significativas, adaptándose al ritmo de los estudiantes y conectando con sus ideas previas (De la Calle, 2016). En este sentido, cabe matizar que estudios como los de Sánchez (2015) y Vega (2015) señalan que en los centros donde se lleva a cabo esta metodología han mejorado los resultados académicos.

Y así lo hemos podido comprobar en los grupos de discusión discentes. En las anotaciones que se recogen a continuación se muestra cómo los conocimientos adquiridos en sus respectivas investigaciones saben integrarlos en su realidad cuando les ponemos en supuestos prácticos que tienen que resolver.

Investigadora: ¿Qué contenidos consideras que te ayudan a formarte como ciudadano?

Alumna: Lo del velo.

Alumna: $Y$ lo que dije antes del machismo, que nos ayuda a no serlo. (G.3-4 Quotation 3:42)

Investigadora: De manera similar, el traje de novia actual también cubre bastante el cuerpo de la mujer y también se usa el velo, ¿qué opináis al respecto? ¿este velo también puede significar sumisión respecto al marido?

Alumna: Sí, es verdad que a la iglesia no se puede ir con falda corta. Mi madre me lo dice.

Alumna: Que sigue habiendo machismo porque si somos iguales el velo es sumisión.

Alumna: Investigando los velos y eso, lei que, si no llevas velo o no entras cubierta a la iglesia, puede significar deshonra.

Investigadora: ¿ Y conocer esta información hace que vosotras os planteéis si usar velo o no en un futuro?

Alumna: Sabiendo esto, yo no lo voy a usar.

Alumna: A lo mejor por no salirse del tiesto, por no desentonar, habrá gente que siga llevando el velo. Pero yo no. (G.3-4 Quotation 3:38)

Las salidas o visitas a elementos patrimoniales también tienen presencia en la praxis del docente, siempre contextualizados con el diseño didáctico y con los contenidos curriculares. Por ejemplo, en el caso del grupo de la Sevilla islámica, utilizará su trabajo para hacer de guías en la visita que tenían programada al final curso.

Alumna: Primero visitamos uno de los monumentos más importantes como la Giralda, los Reales Alcázares... 
Profesor: Muy bien, muy bien. Está muy chulo. Estaría bien que en el propio plano hicierais el recorrido, ¿no?

Alumno: ¿̇Poner un plano aparte?

Profesor: El mismo que habéis utilizado antes. Iríamos por aquí... tiramos por acá... Eso estaría chulo. (O5 Quotation: 5:35)

Por todo ello, esta tercera fórmula es especialmente significativa en un profesor posmoderno. Los datos recogidos nos hacen defender el Aprendizaje Basado en Proyectos como la metodología de enseñanza/aprendizaje más idóneo para llegar a ser este tipo de docente, pues como Vallés Villanueva y Vayreda (2008) señalan, es fundamental que las prácticas y las acciones educativas se formalicen en experiencias colectivas e innovadoras, donde la investigación, la experimentación, el diálogo y el intercambio de ideas permitan unas prácticas dinámicas e interactivas.

\subsection{Educación patrimonial y participación ciudadana}

En relación el con problema central, ¿Qué papel tiene la educación patrimonial en la formación de una ciudadanía crítica y comprometida para el profesorado innovador en Ciencias Sociales, Geografía e Historia?; el profesor posmoderno de Ciencias Sociales, Geografía e Historia parte de una didáctica del patrimonio que facilita en el alumnado la creación de una identidad individual pero también colectiva, asumiendo los conocimientos, comportamientos y actitudes propios de una ciudadanía planetaria, comprometiéndose con su entorno a partir de la conservación y la gestión de los elementos patrimoniales.

Nuestro docente entiende que la identidad es un aspecto fundamental para la formación de una ciudadanía democrática. Asumiendo el modelo integral para enseñar y aprender el patrimonio expuesto por Fontal (2003), el cual se sustenta en una estructura de percepción de círculos concéntricos de identidad que visualizan al patrimonio como un proceso que va desde la valoración de los bienes individuales hasta llegar al patrimonio mundial. Esto supone trabajar a partir de los bienes que nos identifican en lo inmediato, para luego poder proyectarnos hacia el pasado y el futuro que queremos que nos identifique.

Pero para que esto pueda darse, los estudiantes deben aceptar de manera consciente y voluntaria esos bienes, ya que "sin dicha aceptación, el legatario es incapaz de reconocer su herencia como un cuerpo integrado que le pertenece, mientras que, al aceptarlo, reconoce en dichos bienes, elementos constitutivos, signos y señas, de su identidad" (Cantón y González Sáez, 2009, p.2); porque, en esencia, sólo en el reconocimiento de tu propia identidad se puede reconocer y comprender el valor de lo ajeno.

\footnotetext{
Profesor: Vale, yo creo que ese es el primer objetivo. Por medio de este trabajo vamos a intentar que conozcáis qué representa los diferentes elementos de nuestro escudo, que simbolizan pues... a nuestro país y brevemente su historia. (O6 Quotation 6:8)
}

Sin embargo, un profesor posmoderno va un paso más allá, enseñando a su alumnado a gestionar su patrimonio independientemente de la labor que hacen las instituciones. Asumir la tarea de la gestión patrimonial implica inevitablemente convertirte en un ciudadano crítico y participativo. De manera que, mediante el planteamiento de problemáticas patrimoniales, conectados con la realidad de los estudiantes, éstos tienen que posicionarse y asumir comportamientos propios de una ciudadanía participativa.

Profesor: Lo que quiero deciros es que de aquí a 200 años puede que la torre Pelli forme parte del patrimonio histórico-artístico de Sevilla, ¿no? $Y$ haya mucha gente que la considere símbolo... Preciosa, interesantísima... 
Los turistas no sólo vienen a ver la Giralda si no también esta otra... eso no lo sabemos.

Pero la idea de lo que es patrimonio puede variar $\dot{¿}$ De acuerdo?

A lo largo de la historia se han derribado muchos edificios que hoy consideraríamos pues... un delito. Sin embargo, se tiraron porque en aquel momento no se valoraban. (O5 Quotation 5:26)

\subsection{Obstáculos encontrados}

En relación a la pregunta, "¿Qué obstáculos de enseñanza/aprendizaje existen para el profesorado innovador en la formación de ciudadanos críticos y participativos a través de la educación patrimonial en Ciencias Sociales, Geografía e Historia?"; a pesar de que el profesor posmoderno cumpla con todos los postulados expuestos hasta ahora y que garantizan una buena praxis, van a existir siempre condicionantes externos que influyen en su práctica docente y obstaculizan el proceso de enseñanza/aprendizaje.

Este problema pudimos abordarlo desde la reflexión final del docente y desde el pensamiento discente tras la praxis. De esta forma, constatamos que, a pesar de la concepción holística y simbólico-identitaria que tiene el docente y que proyectaba en su praxis, los estudiantes, en sus respectivos trabajos de investigación, elegían los elementos patrimoniales basándose en criterios de antigüedad, al ser los más conocidos o ser los que mayor interés turístico despiertan. Del mismo modo, cuando en los grupos de discusión les preguntamos qué principios tenía que cumplir un bien para ser considerado patrimonio, aludían al prestigio, ser conocidos o pertenecer a épocas pasadas.

Investigadora: ¿̇Entonces qué es el patrimonio para vosotros?

Alumna: Algo importante.

Investigadora: ¿¿Pero importante en base a qué? ¿Por qué algo es importante?

Alumna: Porque es conocido... tiene su historia.

Investigadora: Tienes que ser conocido, tiene que tener historia... ¿̇qué más requisitos tiene que tener algo para ser patrimonio?

Alumna: Ya está.(G.2 Quotation 2:9)

La explicación que da el docente a esto, tiene que ver con las ideas previas del alumnado. A su parecer, están basadas en los medios de comunicación y en la cultura dominante. Cultura dominante que, en el caso del contexto escolar, responde a un currículo que limita en cuestiones patrimoniales.

Pues evidentemente es por la cultura dominante, por los medios de comunicación principalmente. (E2 Quotation 1:1)

El currículum limita también. Eso sin ningún género de dudas y .. ¡ vaya! ¡sin duda! ¿Por qué? Porque siempre existen una serie de constricciones y el currículo es constrictivo, querámoslo o no. (E2 Quotation 1:17)

Por otro lado, nos llamó mucho la atención que los estudiantes diferenciaran entre dos tipos de patrimonio: el institucionalizado o el que está recogido en los libros de textos; y el patrimonio no institucionalizado, es decir, el patrimonio local y más cercano.

Alumna: Es que, para mí, hay dos tipos de significados para decir que esto es patrimonio. Por ejemplo, el patrimonio que nosotros decimos que es patrimonio porque es mundialmente conocido o que es turístico. Pero lo otro... el otro patrimonio ha sido el que ha marcado un antes y un después en nuestros pasados y que han hecho una cosa importante para... nosotros. (G.1. Quotation 1:15) 
Para nuestro docente, esta doble clasificación se debe a que los alumnos distinguen entre el patrimonio escolar, es decir un concepto de patrimonio reduccionista de carácter histórico-artístico, vinculado a una perspectiva disciplinar y academicista y con escasas propuestas participativas en relación con la enseñanza del mismo. Este tipo de patrimonio lo podemos vincular con las perspectivas monumentalista, excepcionalista e histórica, que son predominantes, como vimos anteriormente, en los criterios de elección de los discentes a la hora de abordar sus temas de investigación. Y el patrimonio vivido, es decir, el que forma parte de la vida familiar de los estudiantes y que se asocia con las tradiciones y las fiestas de la localidad.

Bueno ahí, yo creo que lo que habría que considerar un poco es la diferenciación entre
lo escolar y lo no escolar. Me parece a mí que lo que ellos vinculan más con patrimonio
histórico-artístico, como patrimonio de la humanidad... está más vinculado a lo
escolar, a los conocimientos adquiridos en la escuela; mientras que lo que tienen que
ver con el patrimonio más identitario, más de... material e inmaterial, más con lo
propio, con lo local. (E2 Quotation $1: 9$ )

Por último, cuando preguntamos al docente acerca de los obstáculos que encuentra y que impiden que sus concepciones del patrimonio simbólico-identitario se trasladen a la realidad del aula con referentes concretos, pudiéndose romper así con estas distinciones; alude que es su propia formación docente la que en la mayoría de ocasiones limita su praxis: "Mi propia formación profesional también me limita. Yo no caigo en todo. Uno sigue aprendiendo" (E2 Quotation 1:21). Otra cuestión que dice limitarle en su praxis es la existencia de trabas burocráticas, como es el currículo oficial y el carácter impositivo que éste tiene en determinados aspectos conceptuales, aunque no metodológicos. Afirma sentirse en un debate personal entre lo que la ley impone y lo que él, como profesional, entiende que tiene que hacer.

Dificultades, muchas. Ya hemos hablado de currículo. Aun cuando uno trata de...
impugnar, como diría... sería la palabra "impugnar", ¿no? Impugnar relativamente.
¿Por qué? Porque también te sientes como funcionario... El hábito de funcionario es
intentar cumplir con la ley. Y la ley impone. Ya te digo que la orden del 20 de agosto
de 2007, por ejemplo, era muy flexible, pero al final es subsidiaria del real decreto y
todo lo demás. Entonces el currículo constriñe. Constriñe la familia. Constriñe la
inspección. Relativamente... porque luego tú dices, yo estoy trabajando competencias,
eh... cuidado... mi forma de trabajar, metodológicamente al menos, ¿̇no?, está más
próximo a lo que dice la ley. Ahí tengo si quieres un pequeño debate personal. Y luego
está el asunto de... ya lo viviste... a mí me riñen los profesores porque como ellos
trabajan moviendo las mesas, hay profesores que se me quejan, se me quejan... eso
también constriñe. (E.2 Quotation 1:19)

Pero el obstáculo más significativo que dice tener en el desarrollo de su docencia es la falta de motivación y el escaso interés que muestran algunos estudiantes, a pesar de tener siempre en cuenta sus ideas previas y sus motivaciones. Afirma que no sabe cómo estimularles para que puedan sentir emoción en el aprendizaje, de manera que puedan desarrollar estos vínculos de identidad, que comentamos a lo largo de todo este estudio, y que son necesarios para alcanzar una formación de ciudadanía democrática.

\footnotetext{
También veo que soy incapaz de conseguir que todos los del grupo se motiven por igual. Eso lo noto muchísimo. $Y$ siempre hay alumnos que algo hacen... no sé qué, no sé cuántos... Y claro el grupo de cuatro o cinco... Unos tienen un ocho y otros tienen un seis, ¿̇no? Porque he sido incapaz de conseguir... ¿̇sabes? Porque más allá de que tengan más o menos capacidades, ¿̇no? El asunto es que yo lo veo que no... no, no, no... están identificados con aquello o por lo menos no manifiesta emoción, no manifiesta interés. (E.2 Quotation 1:33)
} 
En definitiva, se pone de manifiesto la importancia del carácter crítico que tiene el docente posmoderno, no solo hacia la sociedad de la que forma parte, sino también hacia sí mismo y su praxis.

\section{Conclusiones}

Para concluir, queremos señalar que se trata de un estudio novedoso en este campo, pues la escasez de estudios previos que conectan educación patrimonial y ciudadanía así lo atestiguan; siendo a su vez esta novedad, la limitación principal con la que nos encontramos para poder desarrollar una discusión más amplia y productiva en este sentido. Aun así, podemos extraer conclusiones relevantes, así como dejar constancia de la necesidad de establecer futuras líneas de trabajo.

A nivel práctico, es importante destacar que las relaciones entre el conocimiento y la acción por parte del profesorado son posibles, pero éstas suelen ser difusas, complejas y complicadas, exigiendo un esfuerzo al profesional docente. Además, las relaciones teoríapráctica van ligadas al propio desarrollo profesional, a la experiencia, al contexto de trabajo, intereses personales, etc. De forma que, a partir del análisis de este profesor, es posible extraer algunas líneas a seguir respecto a cómo el profesorado en general puede superar muchas de las limitaciones de la revisión bibliográfica que hicimos al inicio de nuestra investigación. Entre otras destacamos de este docente, la autoformación, la autocrítica, la innovación y el compromiso con la propia coherencia. Todo ello, exige un esfuerzo permanente, cuidar y cultivar el pensamiento y la acción y un análisis de ambos para valorar lo más objetivamente posible el propio desenvolvimiento profesional.

Respecto a la conceptualización de patrimonio, el docente innovador del caso de estudio entiende el patrimonio desde una perspectiva simbólico-identitaria y desde un planteamiento holístico. Por otro lado, dada la importancia que da la acción, al compromiso y a la búsqueda de la justicia social, el docente asume en su propia praxis aspectos conceptuales de la ciudadanía crítica y de la democracia radical. De forma que, para este profesor, lo más importante de su tarea educativa es que su alumnado desarrolle un pensamiento crítico y participativo, que consigue a través de una metodología activa como es el Aprendizaje Basado en Proyectos. Además, tanto en su praxis como en su pensamiento docente existe una imbricación plena entre el patrimonio, los contenidos de Ciencias Sociales y la realidad socio-natural del alumnado. Todo ello lleva a que los estudiantes puedan construir una identidad individual pero también planetaria a partir del compromiso que establecen con los elementos patrimoniales de su entorno.

No obstante, a pesar de que el docente proyectaba en su praxis la mencionada concepción holística y simbólico-identitaria del patrimonio, en sus trabajos, los estudiantes optaban por seleccionar elementos patrimoniales por criterios de antigüedad y desde un planteamiento reduccionista de carácter histórico-artístico vinculado con las perspectivas monumentalista, excepcionalista e histórica. De este modo, tras el análisis, se concluye que algunos de los obstáculos principales a los que se enfrenta la educación patrimonial y ciudadana responden a la acción de los medios comunicación y la influencia de la cultura dominante. Otra serie de condicionantes detectados en la investigación y que impiden una buena praxis, son los relacionados con su formación docente, concretamente con la necesidad de mantenerse actualizado en temas de patrimonio y de didáctica; así como la 
rigidez burocrática de la institución escolar y la falta de motivación que dice detectar en el alumnado en general al inicio del curso escolar.

Con todo ello, podemos decir que, investigaciones como la que aquí presentamos, ponen de manifiesto que, si el docente de Ciencias Sociales relaciona su formación académica y su praxis, puede dar con fórmulas educativas exitosas para constituirse como un profesional de la educación. Y en esta fórmula educativa, la educación patrimonial es el factor fundamental para formar ciudadanos/as comprometidas con su realidad que, como vimos en la introducción, es el fin último de todo sistema educativo.

Por último, queremos concluir mostrando la importancia de comparar estos datos con nuevos estudios e investigaciones sobre el tema para responder mejor a la pregunta de: ¿qué y cómo enseña el profesorado innovador educación para la ciudadanía utilizando el patrimonio como contenido pedagógico en Ciencias Sociales, Geografía e Historia en Educación Secundaria Obligatoria? Concretamente creemos necesario seguir realizando investigaciones de estudio de casos de docentes innovadores con el fin de poder diseñar un modelo docente en relación a la educación patrimonial y educación para la ciudadanía. Además, sería interesante poder hacer un seguimiento en el tiempo de este docente en cuestión para comprobar si los obstáculos detectados pueden ser salvados con las propuestas que él mismo hacía en la entrevista de reflexión final.

\section{Referencias}

Álvarez, C. (2013). ¿Puede superar el profesorado la quiebra teoría-práctica? Un estudio de caso. REICE. Revista Iberoamericana sobre Calidad, Eficacia y Cambio en Educación, 1 1(4), 109-127.

Ancess, J., Barnett, E. y Allen, D. (2007). Using research to inform the practice of teachers, schools, and school reform organizations. Theory into Practice, 4(4), 325-333. https://doi.org/10.1080/00405840701593915

Arthur, J., Davies, I. y Hahn, C. (2008). The Sage handbook of education for citizenship and democracy. Los Ángeles, CA: Sage Publications.

Barragán Rodríguez, J. M. (2005). Educación artística, perspectivas críticas y práctica educativa. En R. Marín Viadel (Ed.), Investigación en educación artística: Temas, métodos y técnicas de indagación sobre el aprendizaje y la enseñanza de las artes y culturas visuales (pp. 43-80). Granada: Universidad de Granada.

Blömeke, S., Busseb, A., Kaiserb, G., Königc, J. y Suhld, U. (2016). The relation between contentspecific and general teacher knowledge and skills. Teaching and Teacher Education, 56, 3546. https://doi.org/10.1016/j.tate.2016.02.003.

Calaf, R. (2009). Didáctica del patrimonio. Epistemología, metodología y estudios de casos. Gijón: Trea.

Cantón, V. y González Sáez, O. J. (2009). Notas para una aproximación a la educación patrimonial como creadora de identidad y promotora de la calidad educativa. Recuperado de http://www.correodelmaestro.com/anteriores/2009/octubre/2incert161.htm

Cuenca, J. M. (2002). Análisis de concepciones sobre la enseñanza del patrimonio en la educación obligatoria. Enseñanza de las Ciencias Sociales: Revista de Investigación, 2, 37-45.

Cuenca, J. M. (2003). El patrimonio en la didáctica de las ciencias sociales. Análisis de concepciones, dificultades y obstáculos para su integración en la enseñanza obligatoria. Tesis doctoral. Universidad de Huelva. 
De Alba, N. (2007). ¿Qué ciudadanía? ¿Qué educación para la ciudadanía? En R. M. Ávila, R. López Atxurra, y E. Fernández de Larrea (Eds.), Las competencias profesionales para la enseñanzaaprendizaje de las ciencias sociales ante el reto europeo y la globalización (pp. 345-352). Bilbao: Universidad del País Vasco.

De Castro, P. (2016). Cartografía auto etnográfica de una genealogía de programas de educación patrimonial desde la perspectiva del aprendizaje basado en proyectos y la investigación-acción. Tesis doctoral. Universidad de Valladolid.

De La Calle, M. (2016). Aprendizaje basado en proyectos (ABP): Posibilidades y perspectivas en ciencias. Íber. Didáctica de las Ciencias Sociales, 82, 7-12.

Delgado-Algarra, E. J. (2015). Conocimiento glocal y pensamiento crítico en la educación del siglo XXI. International Journal of educational research and Innovation, 4, 1-5.

Delgado-Algarra, E. J. y Estepa, J. (2015). Educación ciudadana y memoria histórica en la enseñanza de las ciencias sociales. Enseñanza de las Ciencias Sociales, 14, 97-109.

Delgado-Algarra, E. J. y Estepa, J. (2016). Ciudadanía y memoria histórica en la enseñanza de la historia: Análisis de la metodología didáctica en un estudio de caso en ESO. Revista de Investigación Educativa, 34(2), 52 1-534. https://doi.org/10.6018/rie.34.2.224891

Delgado-Algarra, E. J. y Estepa, J. (2017). Educación ciudadana y dimensiones de la memoria en la enseñanza de ciencias sociales: Investigación sobre las concepciones del profesorado de educación secundaria de Huelva y provincia. Educación XX1, 20(2), 259-278.

https://doi.org/10.5944/educxx 1.19041

Efland, A. D., Freedman, K. y Stuhr, P. (2003). La educación en el arte posmoderno. Barcelona: Paidós.

Eisner, E. W. (1998). El ojo ilustrado. Indagación cualitativa y mejora de la práctica educativa. Barcelona: Paidós.

Elliot, J. (2010). El estudio de la enseñanza y el aprendizaje: Una forma globalizadora de investigación del profesorado. Revista Interuniversitaria de Formación del Profesorado, 68(24), 201-222.

Escudero, J. M. y Martínez Domínguez, B. (2016). La formación continuada: Precisiones, propuestas y advertencias. Cuadernos de Pedagogía, 469, 48-50.

Estepa, J. (2012). La formación del profesorado para enseñar la participación en el grado de maestro y en el master de profesorado de secundaria. Una alternativa basada en los problemas prácticos profesionales. En N. De Alba, F. F. García Pérez y A. Santiesteban (Eds.), Educar para la participación ciudadana en la enseñanza de las ciencias sociales (pp. 211-220). Sevilla: Díada Editora.

Estepa, J. (2013) (Ed.) La educación patrimonial en la escuela y el museo: Investigación y experiencias. Huelva: Servicio de Publicaciones de la Universidad de Huelva.

Estepa, J., Wamba, A. y Jiménez, R. (2005). Fundamentos para una enseñanza y difusión del patrimonio desde una perspectiva integradora de las ciencias sociales y experimentales. Investigación en la Escuela, 56, 19-26.

Eurydice. (2012). La educación para la ciudadanía en Europa. Madrid: Consejo de Europa.

Fontal, O. (2003). La educación patrimonial. Teoría y práctica en el aula, en el museo y en internet. Gijón: Trea.

Fontal, O. (2006). Claves del patrimonio cultural del presente y desde el presente para abordar su enseñanza. Pulso, 29, 9-31.

Fontal, O. (2008). La importancia de la dimensión humana en la didáctica del patrimonio. En S. M. Mateos (Ed.), La comunicación global del patrimonio cultural (pp. 53-105). Gijón: Trea. 
Freire, P. (1975). La educación como práctica de la libertad. Ciudad de México: Siglo XXI.

García Pérez, F. F. (2006) ¿Qué retos le plantean al profesor las nuevas realidades sociales que nos envuelven? ¿Qué perfil de profesor se considera necesario para una escuela pública, democrática e inclusiva? Conciencia Social, 10, 40-45.

Lucas, L. (2018). La enseñanza del patrimonio y de la ciudadanía en las clases de Ciencias Sociales. Un estudio de caso en ESO. Tesis doctoral. Universidad de Huelva.

Marí Ytarte, R. M, Moreno, R. e Hipólito, N. (2016). Educación y ciudadanía. Propuestas educativas desde la controversia. Foro de Educación, 14(20), 49-69. https://doi.org/10.14516/fde.2016.014.020.005

Martín Cáceres, M. (2012). La educación y la comunicación patrimonial: Una mirada desde el museo de Huelva. Tesis doctoral. Universidad de Huelva.

Martínez Rodríguez, R. (2013). Profesores entre la historia y la memoria. Un estudio sobre la enseñanza de la transición dictadura-democracia en España. Tesis doctoral. Universidad de Valladolid.

Matozzi, I. y Ávila, R. M. (2009). La didáctica del patrimonio y la educación para la ciudadanía. En R. Avila, B. Borghi y M. Matozzi (Eds.), Léducaziones alla cittadinanza europea e la formazione degli insegnanti. Un progetto educativo per la "strategia di Lisbona (pp. 327-352). Bolonia: Pàtron Editore.

Molina Girón, L. A. (2013). ¿Cómo la escuela educa para una ciudadanía activa? Una experiencia de educación cívica ciudadana en Canadá. Multidisciplinary Journal of Educational Research, 3, 296-326.

Monteagudo, J. y Oliveros, C. (2016). La didáctica del patrimonio en las aulas. Un análisis de las prácticas docentes. Universidad, Escuela y Sociedad, 1, 64-79.

Navarro, E. (2012). La enseñanza de la historia de España y el desarrollo de las competencias ciudadanas. El conocimiento del alumnado al finalizar el Bachillerato. Tesis doctoral. Universidad de Murcia.

Páramo, D. (2015). La teoría fundamentada (grounded theory), metodología cualitativa de investigación científica. Pensamiento y Gestión, 39, 119-146.

Porlán, R. y Rivero, A. (1998) El conocimiento de los profesores. Sevilla: Diada.

Rathgen, E. (2006). In the voice of teachers: the promise and challenger of participating in classroom-based research for teachers professional learning. Teaching and Teacher Education, 22(5), 580-591. https://doi.org/10.1016/j.tate.2006.01.004

Rossman, G. B. y Rallis, S. F. (1989). Learning in the field. An introduction to qualitative research. Londres: Sage Publications.

Sánchez, J. (2015). Qué dicen los estudios sobre el aprendizaje basado en proyectos. Recuperado de http://actualidadpedagogica.com/estudios_abp/

Schugurensky, D. (marzo, 2012). El profesorado y la enseñanza de la educación ciudadana. Comunicación presentada en el XXIII Simposio Internacional sobre la Didáctica de las Ciencias Sociales. Universidad de Sevilla.

Schulz, W. (2012). Educación para la ciudadanía y participación ciudadana. Una presentación del estudio ICCS 2009 y sus resultados. En N. De Alba, F. F. García y A. Santisteban (Eds.), Educar para la participación ciudadana en la enseñanza de las ciencias sociales (pp. 47-63). Sevilla: AUPDCS.

Sobejano, M. J. y Torres, P.A. (2010). Enseñanza de la historia en secundaria. Historia para el presente y educación ciudadana. Madrid: Tecnos. 
Vallés Villanueva, J. y Vayreda, M. (2008). Experiencias en la educación del patrimonio. Construyendo puentes para un futuro inclusivo. En M. Martín (Ed.), Patrimonio y sociedad (pp. 69-85). Pamplona: Cederna-Garalur.

Vega, V. (2015). Project-based learning research review. Recuperado de: www.edutopia.org/pblresearch-learning-outcomes

\section{Breve CV de los autores}

\section{Laura Lucas}

Doctora y profesora del Dpto. de Didáctica de las Ciencias Sociales de la Facultad de Educación del campus de Palencia (Uva). Miembro de la cátedra de Género en la Universidad de Valladolid e integrante del grupo de investigación DESYM y Red 14. También pertenece al grupo Arte, Investigación y Feminismo de la Universidad del País Vasco (EHU/UP). Su principal línea de investigación se centra en el Patrimonio en femenino para la formación de una ciudadanía igualitaria en la Didáctica de las Ciencias Sociales. Recientemente ha iniciado una segunda tesis doctoral en $\mathrm{H}^{\mathrm{a}}$ del Arte relacionada con los Estudios sobre las Mujeres y de Género en el Arte Contemporáneo. ORCID ID: https://orcid.org/oo00-0002-7214-181X. Email: laura.lucas.palacios@uva.es

\section{Emilio Delgado-Algarra}

Profesor del departamento de Didácticas Integradas de la Universidad de Huelva (España) y doctor en Investigación en Enseñanza y Aprendizaje de las Ciencias Sociales (UHU, España). Investigador en Red14 y DESYM "Didáctica de las Ciencias Experimentales, Sociales y Matemáticas". Director del Centro Académico y Cultural de Asia Oriental UHU (España). Participación activa en numerosas conferencias internacionales, comités científicos y organizadores, proyectos culturales, proyectos de investigación y publicaciones sobre enseñanza de las ciencias sociales y de la historia, educación ciudadana, memoria histórica; incluyendo trabajos sobre competencia plurilingüe y pluricultural, investigación de acción y educación comparada con Japón. ORCID ID: https://orcid.org/OOOO-O002-2183-8465. Email: emilio.delgado@ddcc.uhu.es 